\title{
A Greenland Sea Perspective on the Dynamics of Postconvective Eddies*
}

\author{
K. I. C. OLIVER ${ }^{+}$ \\ School of Environmental Sciences, University of East Anglia, Norwich, United Kingdom
}

T. ELDEVIK

Nansen Environmental and Remote Sensing Centre, and Bjerknes Centre for Climate Research, Bergen, Norway

D. P. STEVens

School of Mathematics, University of East Anglia, Norwich, United Kingdom

A. J. WATSON

School of Environmental Sciences, University of East Anglia, Norwich, United Kingdom

(Manuscript received 16 May 2007, in final form 26 May 2008)

\begin{abstract}
Open ocean deep postconvection contributes to the formation of the dense waters that fill the global deep ocean. The dynamics of postconvective vortices are key to understanding the role of convection in ocean circulation. Submesoscale coherent vortices (SCVs) observed in convective regions are likely to be the anticyclonic components of hetons. Hetons are dipoles, consisting of a surface cyclone and a weakly stratified subsurface anticyclone, that can be formed by convection. Here, key postconvective processes are investigated using numerical experiments of increasing sophistication with two primary goals: 1) to understand how the ambient hydrography and topography influence the propagation of hetons and 2) to provide a theoretical context for recent observations of SCVs in the Greenland Sea.

It is found that the alignment of hetons is controlled by ambient horizontal density gradients and that hetons self-propagate into lighter waters as a result. This provides a mechanism for transporting convected water out of a cyclonic gyre, but the propagation is arrested if the heton meets large-amplitude topography. Upon interaction with topography, hetons usually separate, and the surface cyclone returns toward denser water. The anticyclone usually remains close to topography and may become trapped for several hundred days. These findings may explain the observed accumulation and longevity of SCVs at the Greenland Fracture Zone, on the rim of the Greenland Sea gyre. The separation and sorting of cyclones from anticyclones have likely implications for the density and vorticity budgets of convective regions.
\end{abstract}

\section{Introduction}

Convection in the marginal seas of the North Atlantic Ocean has long been considered a key process in forming the dense waters that fill much of the global

\footnotetext{
* Bjerknes Centre for Climate Research Publication Number
} A193.

+ Current affiliation: Department of Earth and Environmental Sciences, The Open University, Milton Keynes, United Kingdom.

Corresponding author address: K. I. C. Oliver, Department of Earth and Environmental Sciences, The Open University, Walton Hall, Milton Keynes MK7 6AA, United Kingdom.

E-mail: k.i.c.oliver@open.ac.uk deep ocean. Overflow waters across the GreenlandScotland Ridge, feeding North Atlantic Deep Water, are formed through a combination of rapid heat loss in the Norwegian Sea, brine rejection through ice formation, and open ocean convection. It is now thought that only a modest component of overflow water is formed directly by deep convection in the Greenland Sea (Mauritzen 1996; Watson et al. 1999; Olsson et al. 2005). However, the densest water in the Nordic seas is formed by Greenland Sea convection, and changes in deep convection have been linked to changes in the large-scale dense water properties of the region on multidecadal time scales (Bönisch et al. 1997). These waters are too dense to overflow the Greenland-Scotland Ridge until they mix with lighter waters aloft, and therefore provide a lower boundary condition on inter- 


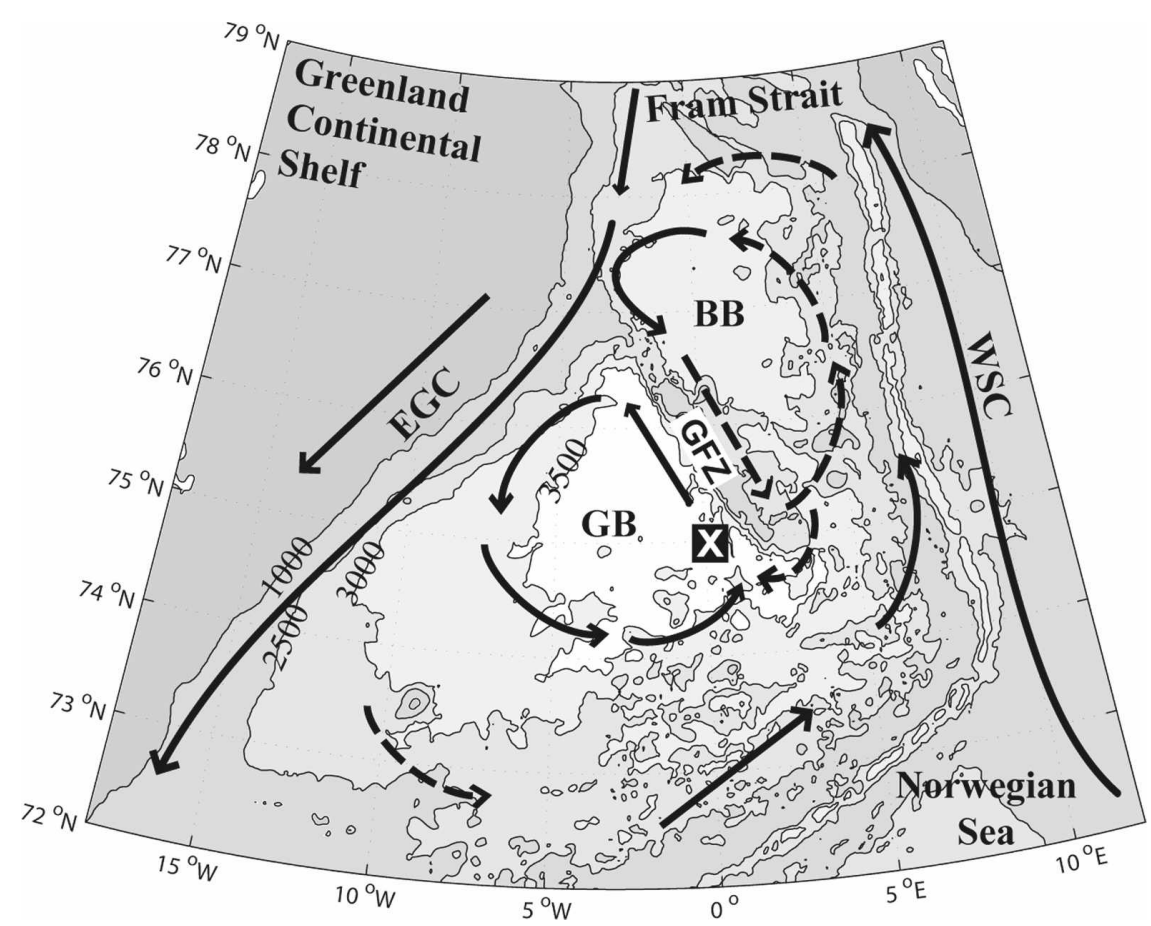

FIG. 1. Topography and schematic of the mean intermediate depth circulation of the Greenland Sea and its environs. Marked isobaths are 1000, 2500, 3000, and $3500 \mathrm{~m}$. Marked features are Greenland Basin (GB); Boreas Basin (BB); Greenland Fracture Zone (GFZ); East Greenland Current (EGC); West Spitsbergen Current (WSC); location where long-lived stationary SCVs have been observed (X) (Gascard et al. 2002; Wadhams et al. 2004). Dashed arrows indicate currents that are either less well established by observations or less permanent. Studies on which the schematic is based include results from a tracer release experiment (Messias et al. 2008), observations from floats (Poulain et al. 1996; Gascard et al. 2002; Jakobsen et al. 2003), moored and lowered current meters (Woodgate et al. 1999; Fahrbach et al. 2001; Oliver and Heywood 2003), hydrographic observations (Quadfasel and Meincke 1987), and modeled reconstructions of the circulation of the Nordic seas (Nøst and Isachsen 2003; Eldevik et al. 2005).

mediate waters of overflow density. Thus, the influence of the Greenland Sea on the larger-scale circulation is likely to be on multidecadal time scales.

Understanding convective regions depends on the understanding of postconvective dynamics. It is unusual to observe ongoing deep convection, and the majority of evidence for convection is obtained from observing postconvective vortices (Kasajima et al. 2006), or regional-scale stratification (Ronski and Budeus 2005), weeks or months after convection has ceased. Moreover, the postconvective behavior of vortices is likely to be a key control on the heat and salinity budgets of convective regions (Legg et al. 1996). Postconvective dynamics have been the focus of previous process studies (e.g., Legg and Marshall 1998; Gryanik et al. 2000), but these have been in the context of a uniform ambient hydrography. In practice, convection occurs in cyclonic gyres with significant horizontal density gradients and bounded by steep topography, each of which has the potential to influence the behavior of postconvective vortices.

The topography and mean circulation of the Greenland Sea is plotted in Fig. 1. The Greenland Sea is flanked by land or shallow $(<2500 \mathrm{~m})$ topography on all sides. Warm saline Atlantic water is carried northward to the east of the Greenland Sea in the West Spitsbergen Current, recirculates in the Fram Strait and the Arctic Ocean, and flows southward as part of the East Greenland Current (EGC). The EGC also carries cold freshwaters of polar origin. This large-scale cyclonic circulation around the Nordic seas is ultimately wind driven with a strong barotropic component (Furevik and Nilsen 2005), but there is also significant vertical shear in the flow; for example, the transport along the Arctic front to the southeast is dominated by its baroclinic component (Oliver and Heywood 2003). The Greenland Sea itself consists of the Greenland Basin and the Boreas Basin, separated by the Greenland 
(a)

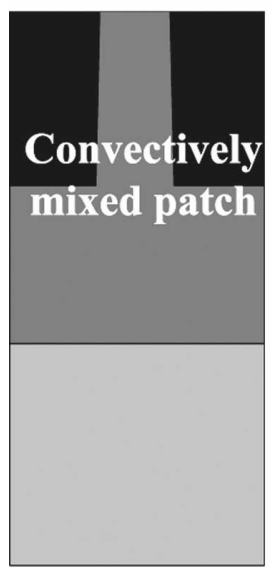

(b)

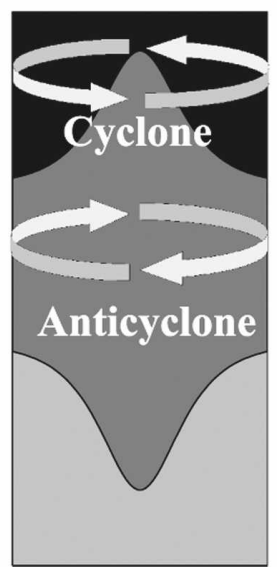

(c)

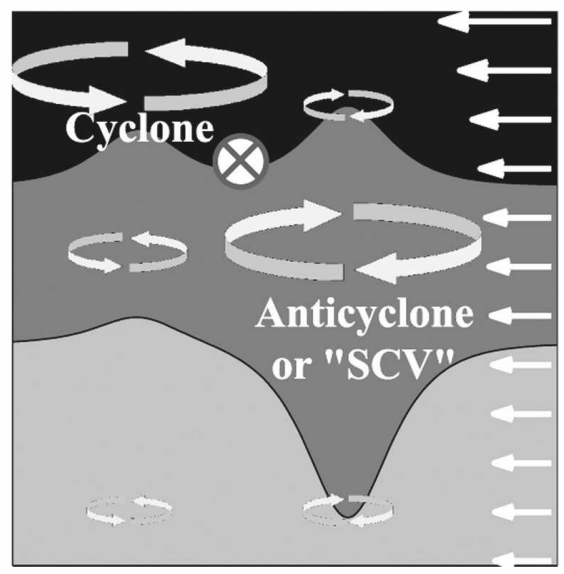

FIG. 2. Vertical section-view schematic of the formation of a heton. Shading represents layers of water defined by density. (a) Surface buoyancy forcing causes local densification of the surface layer, represented here as a column of well-mixed water reaching from the surface to the base of the intermediate water layer. (b) The well-mixed patch sinks. Geostrophic shear results in anticyclonic rotation within the mixed patch and cyclonic rotation aloft. (c) The cyclone and anticyclone separate, forming a baroclinic dipole, or heton. SCVs in convective regions are anticyclones thought to be formed in this way. The heton propagates in the direction of flow between the cyclone and the anticyclone (into the page, in this example). Arrows indicate a possible control on the alignment of hetons: we argue in section 3 that the alignment, and therefore the propagation, of hetons is controlled by the vertical velocity shear resulting from ambient density gradients.

Fracture Zone (GFZ). The Greenland Sea gyre refers to the cyclonic circulation in the Greenland Basin, and this has usually been the focus of studies of Greenland Sea convection, but deep convection has also been observed to occur in the Boreas Basin (Johannessen et al. 2005). There is topographically steered cyclonic circulation in each basin (Jakobsen et al. 2003), and waters of polar and Atlantic origins are observed over the GFZ as a result (Quadfasel and Meincke 1987). This wind-driven circulation leads to the doming of isopycnals toward the gyre center, generating horizontal density gradients. Weak stratification at the gyre center and baroclinic instability in the rim current have long been viewed as the properties of cyclonic gyres that favor deep convection (Killworth 1979).

Rapidly rotating lenses of weakly stratified water have been observed in the Greenland Sea (Gascard et al. 2002; Wadhams et al. 2004; Budéus et al. 2004; Kasajima et al. 2006; Ronski and Budeus 2006) as well as in the Labrador (Clark 1983; Pickart et al. 1996; Lilly et al. 2003) and Mediterranean Seas (Testor and Gascard 2006). These anticyclones have the defining characteristics of submesoscale coherent vortices (SCVs; McWilliams 1985): they are smaller than mesoscale eddies, have been observed to survive for over a year (Wadhams et al. 2004), and have a velocity maximum at middepths. In convective regions, they are likely to be the anticyclonic components of hetons (see Fig. 2). Hetons are baroclinic dipoles that experiments in laboratory tanks and numerical models suggest are produced by deep convection (Marshall and Schott 1999) or by topographic interactions (Dewar 2002a). (For clarity, in this study we only use the expression "SCV" when discussing observations, and otherwise refer to hetons and their cyclonic and anticyclonic components.) Several Greenland Sea SCVs have been observed on the western flank of the GFZ, and at least two long-lived and near-stationary SCVs have been observed near $75^{\circ} \mathrm{N}$, $0^{\circ}$, west of the southern tip of the GFZ (Gascard et al. 2002; Wadhams et al. 2004; see Fig. 1). This may be interpreted as evidence that convection is localized there, but an alternative hypothesis is that the local topography and hydrography cause postconvective vortices to accumulate and/or survive for longer near the GFZ.

The goals of this study are to improve the understanding of how ambient hydrography and topography influence postconvective vortices and to assess the impact of the dynamics of these vortices on the interpretation of observations from the Greenland Sea. In section 2, we briefly summarize existing dynamical theory of vortices in convective regions and the relevance of this theory to the Greenland Sea. In section 3, the experiments are presented. Channel experiments are used 
to develop an understanding of the interaction of hetons both with ambient flow and topography. More realistic basin experiments are used to assess implications of these results for the Greenland Sea, particularly with regard to the observed distribution of SCVs. Our key findings are summarized in section 4 .

\section{Background to the dynamics of postconvective vortices}

\section{a. Heton formation and propagation}

Open ocean deep convection occurs through highly localized plumes of upwelling and downwelling water. The integrated properties of a convective patch can be characterized in terms of an unstably stratified volume undergoing rapid vertical mixing (Send and Marshall 1995). Numerical experiments suggest that such a mixed patch exchanges buoyancy with its stratified surroundings, both during and after convection, through baroclinic instability and the shedding of hetons (Legg et al. 1996). Figure 2 is a schematic representing the processes that lead to the formation and propagation of a heton. At the instant of formation, a heton is a vertically aligned dipole, consisting of a cyclone directly overlying an anticyclone. This may be understood in terms of the redistribution of potential vorticity (Legg and Marshall 1993) or in terms of geostrophic shear: the weakly stratified heton is a buoyant anomaly at depth, causing anticyclonic shear, and a dense anomaly at the surface, causing cyclonic shear.

Of interest here is the propagation and coherence of hetons that are not subject to ongoing convection, either because surface buoyancy loss has ceased or they have left the convective patch. Hetons self-propagate if they are not vertically aligned. For example, the surface cyclone in Fig. 2c lies to the left of the subsurface anticyclone, causing self-propogation into the page. The name "heton" derives from the heat transport associated with this self-propagation (Hogg and Stommel 1985), and the theory describing the behavior of hetons in a rotating uniformly stratified fluid is well developed (e.g., Griffiths and Hopfinger 1986; Legg and Marshall 1993; Carton 2001). Gryanik et al. (2000) developed a quasigeostrophic theory for isolated hetons, with equal magnitude cyclonic and anticyclonic components, in uniform stratification and zero ambient shear. The heton propagates with velocity:

$$
c_{h}=C(b, h) U_{h},
$$

where $C(b, h)$ is a function of the horizontal vortex separation $b$ (nondimensionalized by the bulk Rossby radius $L_{R}$ ) and the convection depth $h$ (nondimension- alized by the bathymetric depth $H$ ). The velocity scale $U_{h}$ is determined by the strength of the initial mixed patch:

$$
U_{h}=\frac{L_{R}}{T_{h}}=\frac{f^{2} A \overline{g^{\prime}}}{4 \pi H^{2} N^{3}}, \quad L_{R}=\frac{N H}{f}, \quad T_{h}=\frac{4 \pi H^{3} N^{4}}{f^{3} A \overline{g^{\prime}}},
$$

where $f$ is the Coriolis parameter, $N$ is the ambient buoyancy frequency, $T_{h}$ is a time scale, $A$ is the horizontal area of the initial vertically aligned heton, and $\overline{g^{\prime}}$ is the mean buoyancy anomaly in the initial mixed patch, so that $A \overline{g^{\prime}}$ is the total buoyancy anomaly per unit depth. [Gryanik et al. (2000) actually derived $U_{h}$ in terms of a vorticity source rather than a buoyancy anomaly.] For large horizontal separations between the cyclone and the anticyclone, $(b \gg 1), C \sim b^{-1}$; for full details, see Gryanik et al. (2000).

\section{b. Vortex interaction with ambient flow and topography}

The baroclinic rim current around convective patches plays a key role in generating and propagating hetons (Legg and Marshall 1993). Theory and numerical experiments suggest that the density transport across narrow fronts scales with the time-mean alongfront geostrophic velocity and the horizontal density gradient (e.g., Visbeck et al. 1996; Spall and Chapman 1998). On the other hand, Legg and Marshall (1998) found that a larger-scale barotropic cyclonic gyre circulation, such as is generally found in convection regions, stabilizes the frontal region in a heton model. This reduced the efficiency of cross-frontal density transport by heton generation. This experiment has not been repeated for a baroclinic gyre. Walsh and Pratt (1995) derived a pointvortex model in which asymmetric but like-signed point vortices could propagate relative to the ambient flow in the presence of vertical shear; this propagation was in a direction parallel to the ambient flow. However, Kasajima et al. (2006) speculated that vertical shear in the ambient flow contributes to the observed rapid migration of SCVs across the ambient flow in the Greenland Sea, citing tropical cyclones as a similar atmospheric process. Tropical cyclones consist of a surface cyclone and an upper-atmosphere anticyclone. There is evidence that there is propagation to the left (in the Northern Hemisphere) of the ambient vertical velocity shear (Wu and Emanuel 1993): the upper anticyclone is displaced downstream of the lower cyclone, forming a baroclinic dipole which then self-propagates in the direction of flow between the vortices. In section 3, we test the hypothesis that an analogous process acts on hetons. 
There have been many studies of the interaction of vortices with topography in the context of the opposite limiting cases of barotropic and surface-trapped vortices. A barotropic mesoscale vortex over a topographic slope is subject to topographic $\beta$ drift (Carnevale et al. 1991; Flór and Eames 2002), comparable to westward vortex drift on a $\beta$ plane. Over strong slopes, vortices can become stationary and surface trapped due to dispersion at depth in topograhic waves (LaCasce 1998). Surface-trapped vortices that exceed the Rossby radius may be stabilized by steep topography. Hetons are neither barotropic nor surface trapped, with a velocity maximum at middepths in the anticyclone. Jacob et al. (2002) found that analytical theories that successfully predict the propagation of barotropic or monopolar baroclinic vortices break down in the case of counterrotating vortices such as hetons and argued that an experimental approach was needed to understand their behavior. The role of isolated topography in the generation and evolution of hetons was examined by Dewar (2002a). Hetons could survive over weak topography, with sizable propagation rates influenced by topography. In the setting of full-column convection in a quasigeostrophic model, Dewar (2002b) found that heton formation is inhibited by weak slopes, but that steep slopes can repulse anticylones due to advection of ambient potential vorticity across isobaths by the heton. Following partial-column convection, however, slope effects were more disorganized, but even weak slopes were able to slow the dispersal of hetons.

\section{Numerical experiments}

Numerical experiments were designed with the objectives of understanding the effects of ambient horizontal density gradients and bathymetry on the behavior of postconvective vortices, both in a general context and in the Greenland Sea specifically. For this reason, mixed-patch release experiments were carried out in a simple channel setting as well as in basin experiments more closely representing the Greenland Sea. All experiments were carried out with the Massachusetts Institute of Technology general circulation model (MIT$\mathrm{gcm}$; Marshall et al. 1997) on an $f$ plane $\left(f=1.5 \times 10^{-4}\right.$ $\left.\mathrm{s}^{-1}\right)$. The vertical resolution of the 40 levels decreased from $11 \mathrm{~m}$ at the surface to $147 \mathrm{~m}$ at the bottom ( 3750 $\mathrm{m})$, and a very high horizontal resolution of $500 \mathrm{~m} \times$ $500 \mathrm{~m}$ was applied in order to resolve postconvective vortices. The parameterized biharmonic horizontal viscosity was $3 \times 10^{6} \mathrm{~m}^{4} \mathrm{~s}^{-1}$ and the Laplacian horizontal tracer diffusivity was $0.2 \mathrm{~m}^{2} \mathrm{~s}^{-1}$, in each case as low as stability constraints would permit. The vertical viscosity and diffusivity was $1 \times 10^{-5} \mathrm{~m}^{2} \mathrm{~s}^{-1}$. The model was allowed to spin down from a prescribed initial state in geostrophic equilibrium. In the experiments presented here, the energy loss to the system was typically $\sim 10 \%$ in 200 days, so the effect of this spindown is unlikely to qualitatively affect the results. The exclusion of wind and surface buoyancy forcing simplifies the interpretation of results at the expense of neglecting processes that are likely to influence the behavior of vortices. Because no buoyancy forcing was applied and the equation of state was linear, we refer directly to density as the active tracer.

\section{a. Mixed-patch release}

We first describe the release of a mixed patch, common to both channel and basin experiments. Columns of homogeneous water with a radius of 6 grid cells (3 $\mathrm{km})$ were added to an ambient density field by increasing the density above $1300 \mathrm{~m}$ to equal the density at $1300 \mathrm{~m}$. (The initialization of the ambient field is described for channel and basin experiments in sections $3 \mathrm{~b}$ and $3 \mathrm{c}$, respectively.) The depth of $1300 \mathrm{~m}$ approximates the bottom of the well-mixed layer of Greenland Sea Intermediate Water, overlying slightly more stratified water (Naveira Garabato et al. 2004; Ronski and Budeus 2005), observed in the Greenland Sea. The mixed patches represent the integral product of buoyancy-forced convection (Send and Marshall 1995) and were tagged with a tracer. The initial velocity field was not altered to be in geostrophic equilibrium with the mixed patch, implying an assumed time scale for convection of less than $f^{-1}$. While this assumption may not be realistic, the products of mixed-patch releases closely resemble the results of laboratory and numerical model convection experiments.

Figure 3 shows the initial behavior of a released mixed patch from a Greenland Sea Basin experiment with idealized bathymetry [section $3 \mathrm{c}(3)$ ], but it is also representative of other experiments. Snapshots of the velocity, tracer, and density fields at day 0 (mixed-patch release), day 10 , and day 20 are presented. The mixed patch is initially a density anomaly with no velocity signature, but it sinks and forms a heton (comparable to the schematic in Fig. 2). Most of the tracer marking the mixed patch is contained within the weakly stratified deep anticyclone, which is associated with a buoyant anomaly below the center of mass of the tracer. A surface cyclone also forms, associated with the upper ocean dense anomaly. This is consistent with the schematic provided in Fig. 2 and with laboratory and numerical experiments (Narimousa 1998; Marshall and Schott 1999). The cyclone mostly contains water from outside the initial mixed patch. The anticyclone and cyclone separate, and after 20 days they do not hori- 

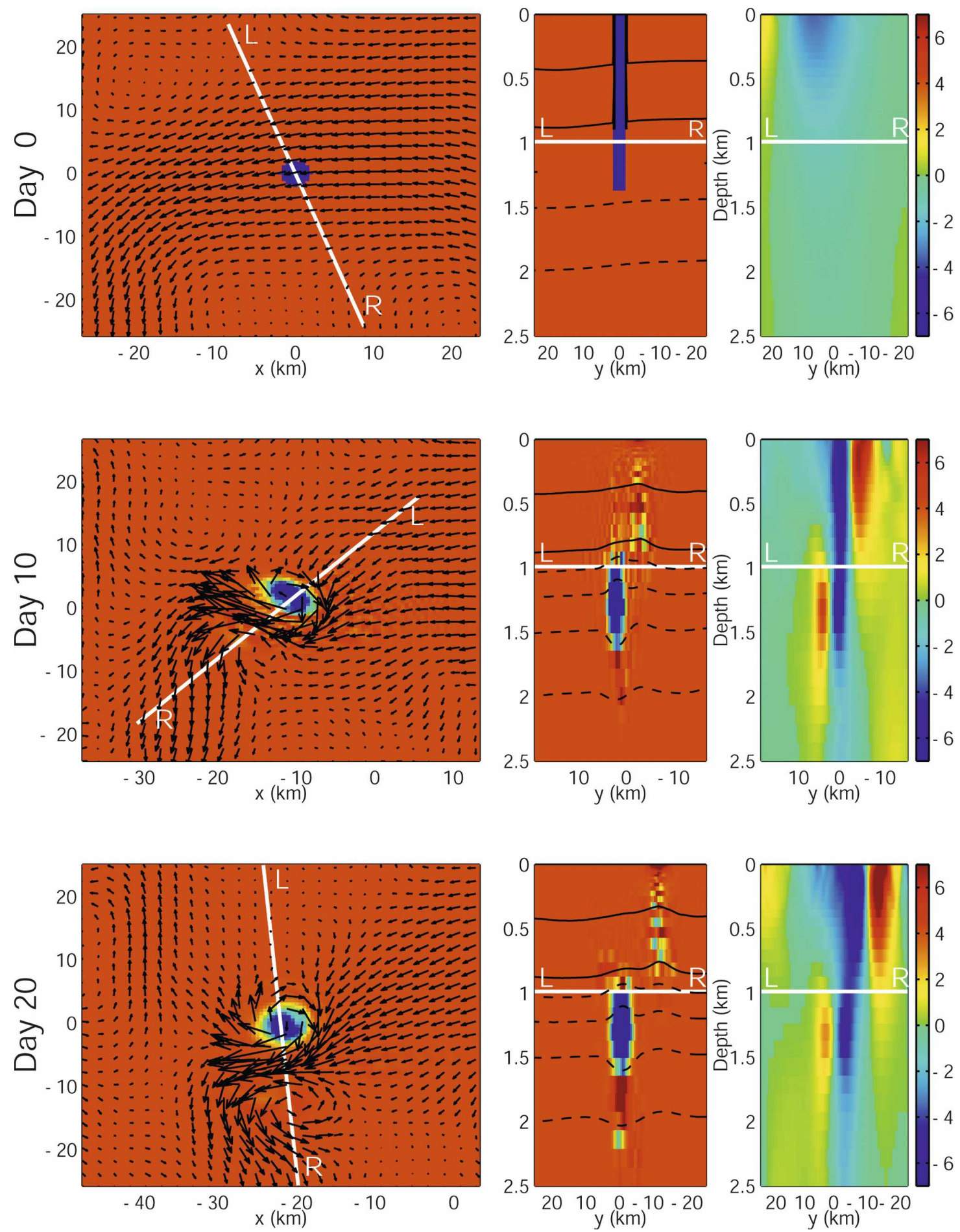

FIG. 3. Time series of the response to the release (day 0) of a mixed patch. (left) A plan view of tracer (marking the initial mixed patch) evolution, with velocity vectors at a spacing is four grid points $(2 \mathrm{~km})$. (middle) Vertical sections of tracer distribution, with density contours (black dashed contour spacing is $0.01 \mathrm{~kg} \mathrm{~m}^{-3}$; separation between the two solid black contours is $0.05 \mathrm{~kg} \mathrm{~m}^{-3}$ ). (right) Cross-section velocity sections (positive flow into the page). The relative position of the plan view and section plots is indicated by white lines. Vertical section alignment is chosen to pass through both the anticyclone and the cyclone. Note that the domain centers move horizontally with the anticyclone center. 


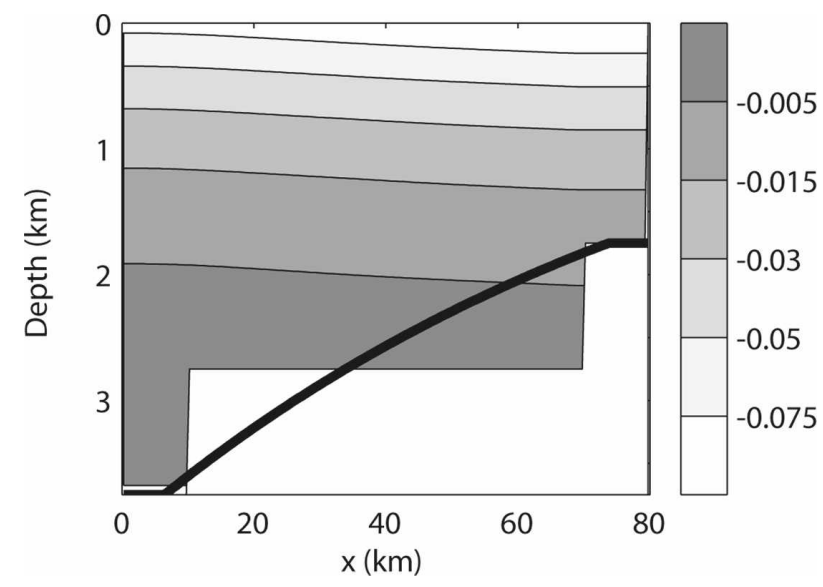

FIG. 4. Section of initial density in the standard experiment. Density is relative to maximum (bottom) value. Bathymetry for flat-bottom experiment is shown; the bold line is the bathymetry for standard sloping-bottom experiment.

zontally overlap. The relative velocities of the anticyclone and cyclone are consistent with separation caused by vertical shear in the ambient flow. Due to ambient horizontal density gradients, the surface cyclone is advected downstream more rapidly than the subsurface anticyclone. The absolute velocity of the heton, however, cannot be explained entirely in terms of advection by the ambient flow. There is a component to the right of the ambient velocity field, which is consistent with heton self-propagation. If the alignment of the heton is set by the ambient density gradients, then the density gradients ultimately control the self-propagation of the heton. Hetons would then be expected to selfpropagate toward lighter water. This line of evidence is developed in section $3 b(2)$.

\section{b. Periodic channel experiments}

\section{1) EXPERIMENT DESIGN}

Idealized experiments were first carried out in an 80 $\mathrm{km} \times 200 \mathrm{~km}$ channel, periodic in the longer dimension. A zonal section of bathymetry and initial hydrography for the standard "flat-bottom" experiments is plotted in Fig. 4. Vertical steps of $1000 \mathrm{~m}$ exist $10 \mathrm{~km}$ from each zonal boundary to provide some topographic stabilization of the flow in the presence of density gradients (Spall 2004); with an entirely flat bottom, eddies rapidly remove the zonal structure in the hydrography. Additionally, Fig. 4 shows bathymetry in "slopingbottom" experiments, where bottom-depth $H$ is described by

$$
\frac{f}{H} \frac{\partial H}{\partial x}=-\beta_{H}, \quad 3750 \mathrm{~m}>H>1700 \mathrm{~m},
$$

where $H(40 \mathrm{~km})=2700 \mathrm{~m}, \beta_{H}$ is the topographic beta associated with barotropic processes, and $H$ is uniform in $y$. In the standard sloping-bottom experiment $\beta_{H}=$ $1.7 \times 10^{-9}(\mathrm{~m} \mathrm{~s})^{-1}$.

Density decreases from west to east at the surface and exponentially decays, with an $e$-folding scale of 700 $\mathrm{m}$, toward a uniform value at infinite depth. Therefore, denser water overlies deeper topography, mimicking convective basins such as the Greenland Sea. Smoothed random perturbations were added to the density field, an example of which can be seen in contours in Fig. 5a, but otherwise the hydrography was meridionally uniform. Over a bathymetric depth of $2700 \mathrm{~m}$, the top-tobottom density difference was $0.095 \mathrm{~kg} \mathrm{~m}^{-3}$. This initial field is consistent with observations of the Greenland Sea (Oliver and Heywood 2003), except for the observed narrow stratified summer surface layer. The initial surface velocity had baroclinic and barotropic components of equal magnitude and direction, which were in geostrophic equilibrium (except for any released mixed patches, as described in section 3a), so that flow was northward along isobaths.

In additional experiments, used to investigate the role of vertical shear strength, initial horizontal density gradients were scaled according to

$$
\begin{aligned}
\rho(x, y, z)= & \rho_{\text {std }}(40 \mathrm{~km}, y, z)+S_{\rho}\left[\rho_{\text {std }}(x, y, z)\right. \\
& \left.-\rho_{\text {std }}(40 \mathrm{~km}, y, z)\right],
\end{aligned}
$$

where $S_{\rho}$ is the scaling factor and $\rho_{\text {std }}$ was obtained from the standard experiment. Additionally, "uniform $N^{2}$ " experiments (which produce unrealistic hydrography including strong density gradients at the bottom) were set up specifically to facilitate comparison with the Gryanik et al. (2000) theory. In these experiments,

$$
\rho(x, y, z)=\rho_{\mathrm{var}}\left(x, y, z_{e}\right)+\left(z-z_{e}\right) \overline{\left(\rho_{z}\right)_{\mathrm{var}}},
$$

where $z_{e}=-500 \mathrm{~m}, \rho_{\mathrm{var}}$ was obtained from the standard experiment (with variable $N^{2}$ ), and $\overline{\left(\rho_{z}\right)_{\mathrm{var}}}$ is the mean vertical density gradient between depths 300 and $1400 \mathrm{~m}$ at $x=40 \mathrm{~km}$ in the standard experiment's initial field. The 300- and 1400-m levels were chosen because these depths approximate the center of cyclones and anticyclones, respectively.

\section{2) Vortex PROPAGATION}

Figure 5 shows snapshots of the density field in the standard flat-bottom experiment with two mixed patches released. Both mixed patches form hetons that are advected to the north by the ambient flow, but also propagate to the east toward lighter water, until approaching steep topography at $x=70 \mathrm{~km}$. Both the cyclone and the anticyclone are visible as distinct fea- 


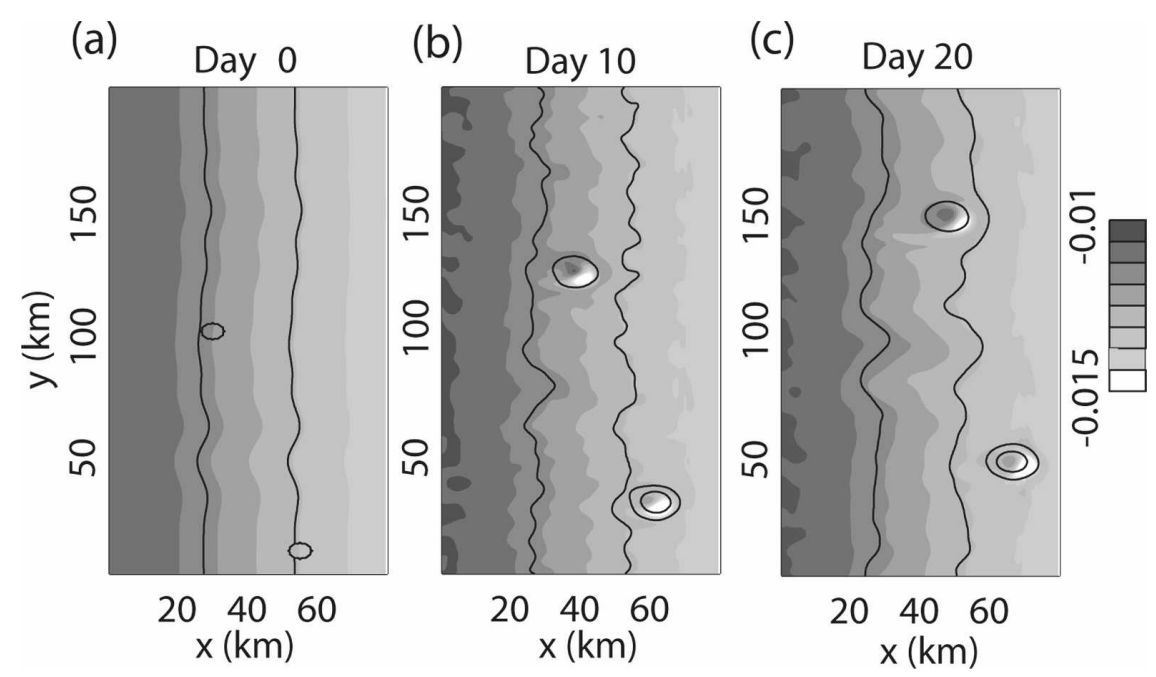

FIG. 5. Density time slices from the early part of a mixed-patch release experiment in the standard flat-bottom channel setup. Density is at $1500 \mathrm{~m}$, relative to the maximum density in the experiment. At this depth the anticyclones are lighter than the ambient water and cyclones are denser than the ambient water. This level is below the maximum depth of the mixed patches before release (at $x=30, y=100$, and $x=55, y=10 \mathrm{~km}$ ). Black contours are for density at $400 \mathrm{~m}$ and depict the upper ocean positive density anomaly associated with both cyclones and anticyclones.

tures, although they propagate together. The cyclones are located to the north and west of the anticyclones, causing heton self-propagation to the east and north. This supports the hypothesized role of ambient hydrography in heton propagation, discussed in section $2 \mathrm{~b}$.

This experiment was repeated under identical conditions, except that the geostrophic shear scaling $S_{\rho}=1$ was replaced by $S_{\rho}=1 / 2,2$, and 4 , respectively. Trajectories of the heton pairs from each experiment are plotted in Fig. 6a. The rate of northward propagation is approximately proportional to the horizontal stratification, simply because the ambient flow is stronger where there is greater geostrophic shear. It is less straightforward to explain why the rate of eastward propagation, prior to meeting topography or heton separation, is also greater in the experiments with stronger shear. The ability of the Gryanik et al. (2000) theory to explain these results is tested using the theoretical eastward propagation rate:

$$
c_{g}=C(b, h) U_{h} \cos \theta,
$$

where $\theta$ is the angle between due north and the vector from the anticyclone to the cyclone. The 10-day mean eastward-propagation rates were compared with propagation rates $c_{g}$ predicted by (6). These are plotted as circles in Fig. $6 \mathrm{~b}$ for the experiment plotted in Fig. $6 \mathrm{a}$ as well as for repeats with different random perturbations in the initial field. Where the heton has met topography, or anticyclones and cyclones have separated, re- sults are not plotted. The $U_{h}$ and $h$ vary little with $S_{\rho}$, so the predicted eastward propagation rate can only be decreased either by increasing the distance between the cyclone and anticyclone (we typically find $b>1$ ) or by rotating the cyclone so it is no longer aligned north of the anticyclone. Rotation is an important source of variability, especially in the $S_{\rho}=1 / 2$ experiment in which westward propagation may even occur due to the cyclone moving south of the anticyclone. This may suggest that the surface cyclone is stronger than the subsurface anticyclone, which would cause cyclonic rotation of a heton (Carton 2001).

Model propagation rates are well correlated $\left(R^{2}>\right.$ 0.6 for each experiment) with predicted rates from Gryanik et al. (2000), but they are systematically slower; however, comparison is inhibited by the variability of stratification in the model. Uniform $N^{2}$ experiments, described in section $3 b(1)$, are used to test the theory more rigorously. (Note, however, that even in these experiments a uniform $N^{2}$ is not preserved as the density field evolves. After 30 days, $N^{2}$ outside hetons has a standard deviation of $\sim 20 \%$.) Again, a good correlation between model and theory is obtained (although $R^{2}=0.48$ for $S_{\rho}=4$ ), but propagation rates in the model are slower. Least squares fitting, with a forced zero intercept, shows that the theory overestimates propagation rate more strongly in experiments with weak horizontal density gradients (Fig. 6b). Thus, while the model qualitatively agrees with the Gryanik 

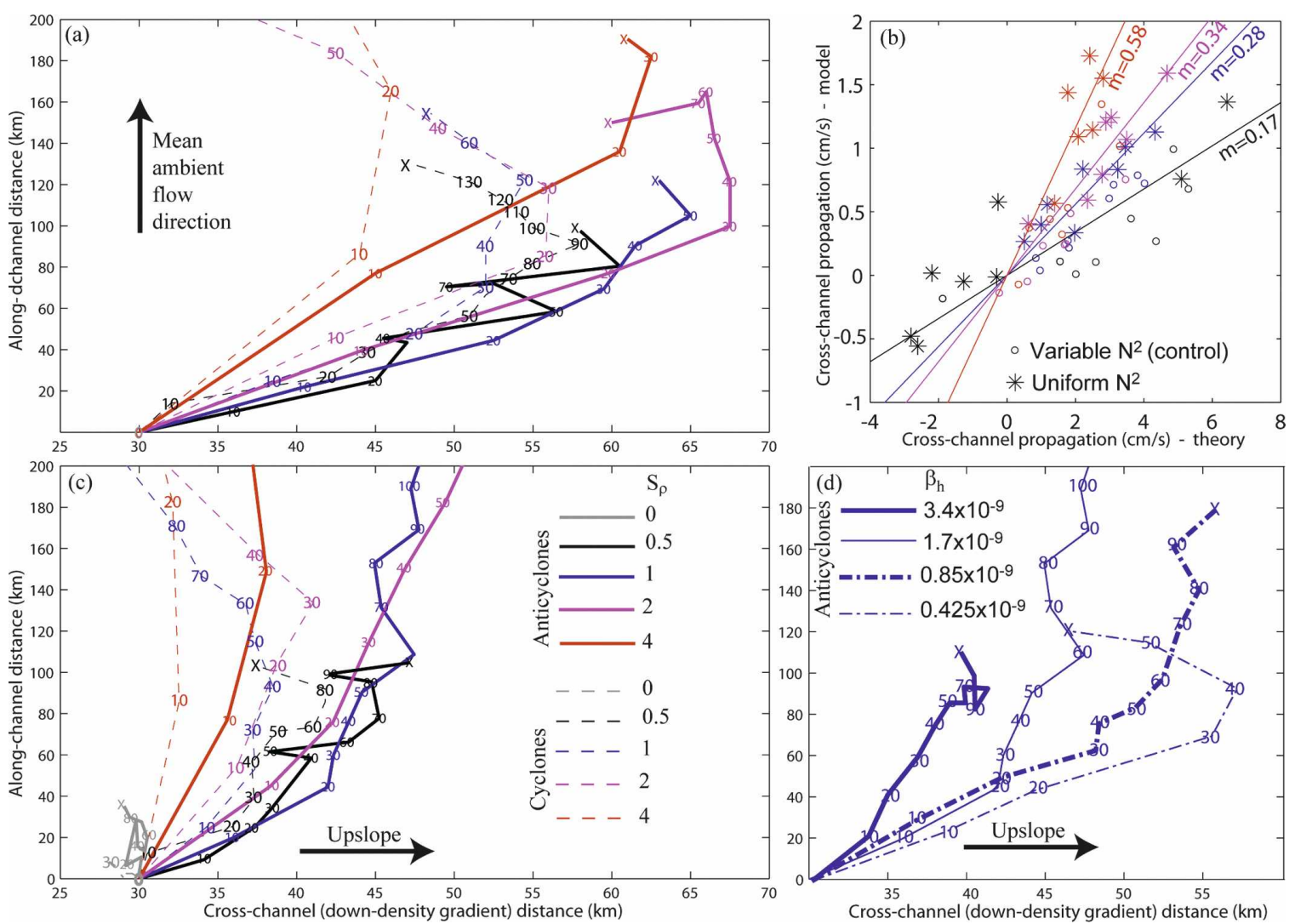

FIG. 6. Trajectories of anticyclones and cyclones in (a) flat-bottom experiments and (c) sloping-bottom experiments $\left(\beta_{H}=1.7 \times\right.$ $\left.10^{-9}\right)$. Trajectories are plotted for experiments where the horizontal stratification is scaled by $S_{\rho}=1 / 2,1,2$, and 4 , respectively, relative to the standard experiment. Numbers indicate the day at which the vortex reached the specified location. An X indicates that the vortex has lost coherence. (b) Model vs theoretical eastward-propagation rates in flat-bottom experiments. Colors indicate different values of $S_{\rho}$, matching those in (a) and (c). Circles are for variable $N^{2}$ experiments, and stars for uniform $N^{2}$ experiments (see text). (d) Trajectories of anticyclones in experiments over different values of slope steepness parameter $\beta_{H}$, with $S_{\rho}=1$.

et al. (2000) theory, the theory is unable to fully explain the relationship between density gradients and propagation rates.

\section{3) The Influence of TOPOGRAPHY}

The behavior of hetons becomes more complex in the presence of topography. In the flat-bottom experiments, the anticyclonic and cyclonic components of the hetons separate upon reaching steep topography at $x=$ $70 \mathrm{~km}$. The cyclones then propagate toward denser water, in some cases eventually reaching a position west of the release point at $x=30 \mathrm{~km}$ (not shown). The anticyclones typically remain close to the topography at $x=$ $70 \mathrm{~km}$ until they lose coherence. The mechanism for the separation of cyclones and anticyclones is explored further in section $3 c(5)$.

Figure $6 \mathrm{c}$ is a repeat of Fig. 6a, but for experiments with a standard sloping bottom [Fig. $4 ; \beta_{H}=1.7 \times 10^{-9}$ $\left.(\mathrm{m} \mathrm{s})^{-1}\right]$. The initial behavior is qualitatively similar to the flat-bottom experiments, but the topography provides a potential vorticity barrier, slowing and eventually arresting eastward propagation. A further effect of sloping topography is the stabilization of the ambient flow (cf. Spall 2004). As a result of the reduced eddy kinetic energy (EKE), the longevity of vortices is typically increased in the presence of sloping topography. Trajectories of a heton in the absence of ambient density gradients, but with a sloping bottom, are also plotted in Fig. 6c. No clear pattern of propagation is found in this case nor in two repeats (not shown) with different initial random perturbations. (Analogous experiments were also run in the flat-bottom configuration, but the heton could remain static and vertically aligned for at least 300 days.)

Several studies (e.g., Dewar 2002b; LaCasce 1998) have obtained qualitatively different vortex behavior, depending on the steepness of the slope. Here, we consider four different slope steepness parameters (in addition to the vertical steps in the flat-bottom experi- 
ments). The range $0.425 \times 10^{-9} \leq \beta_{H} \leq 3.4 \times 10^{-9}$ encompasses the range applied by Dewar (2002b). In Fig. $6 \mathrm{~d}$, the propagation of the anticyclones is plotted for the four different bottom slopes. While the behavior is qualitatively similar in each experiment, the rate of propagation decreases monotonically with slope steepness. In each experiment, eastward propagation is retarded and eventually arrested at a point approximately $10 \mathrm{~km}$ west of the $2000-\mathrm{m}$ isobath. In the $\beta_{H}=0.425 \times$ $10^{-9}$ experiment, the anticyclone then quickly loses coherence, associated with the relatively high ambient EKE due to the weak slope. In the $\beta_{H}=3.4 \times 10^{-9}$ experiment, the anticyclone appears to briefly become trapped shortly after its eastward propagation ceases. Repeat experiments (not shown) show that this does not invariably occur with this domain. This behavior is discussed further in section $3 c(4)$. One difference between our experiments and the experiments of Dewar (2002b) is that Dewar made this comparison under fulldepth convection, whereas our experiments represent partial water column convection. This may explain why we find no qualitative difference in propagation over shallow and steep slopes.

\section{c. Basin experiments}

\section{1) EXPERIMENT DESIGN}

The results of the channel experiments are placed in the context of the Greenland Sea using basin experiments. Two alternative domains were used. In an "idealized bathymetry" experiment, the key topographic features of the Greenland Sea (the Greenland Basin, the Boreas Basin, the GFZ, and the Greenland continental slope) were represented in a simplified form. In a "realistic bathymetry" experiment, 1-km resolution General Bathymetric Chart of the Oceans (GEBCO) bathymetry (IOC et al. 2003) was used. A sloping wall was added at the edge of the domain, except where the Greenland continental slope provides a natural boundary, to provide a closed basin. In both experiments, the $500 \mathrm{~m} \times 500 \mathrm{~m}$ resolution was applied in the central 200 $\mathrm{km} \times 200 \mathrm{~km}$ only, decreasing to $1500 \mathrm{~m} \times 1500 \mathrm{~m}$ resolution at the edge of the domain (see Figs. 7, 10 for the idealized and realistic bathymetry experiments, respectively). The domains were rotated so that Greenland was to the north rather than to the northwest (this does not affect results on an $f$ plane).

Initial densities and barotropic velocities followed bathymetry, using the same approach applied in the sloping-bottom channel experiment [section $3 b(1)$ ]. However, in the basin experiments, the hydrography and velocity fields were smoothed to damp the effect of small-scale topography on the density and velocity fields, and no random perturbation was added. The alignment of density contours and streamlines with topography yielded a cyclonic circulation in the initial field. Unlike the channel experiments, however, the 200-day output from the control experiment was used for mixed-patch release rather than for the initial field: it was found that significant reorganization of the flow occurred within the first 100 days. The mixed-patch population was added in the form of a grid, making the location of each member of the population somewhat arbitrary. In reality, the onset of convection is likely to depend both on the initial stratification and on the local vorticity field. However, the location of convection in the Greenland Sea is not well established, and the application of an arbitrary grid of mixed patches avoids biasing the conclusions of this study.

\section{2) AMBIENT HYDROGRAPHIC AND VELOCITY FIELDS}

In the channel experiments, the key controls of hydrography and topography on heton propagation were established for a highly idealized setting. In this section, we explore the importance of these mechanisms for convective regions such as the Greenland Sea. We begin with a control experiment (without mixed patches), with an idealized representation of the bathymetry in the Greenland Sea. This is preferable to advancing immediately to accurate bathymetry, because the detail of a realistic field may inhibit understanding of the key controls of topography on the simulations. Instead, we represent only those features that we hypothesize to be of major importance to the behavior of postconvective vortices: the Greenland and Boreas Basins, the GFZ, and the Greenland continental slope.

Figure $7 \mathrm{a}$ shows bathymetric contours as well as mean density and velocity fields in the idealized bathymetry simulation. Figure $7 \mathrm{~b}$ shows the corresponding eddy kinetic energy field. Density contours and streamlines approximately follow isobaths, including around the GFZ. (This result does not depend on light water overlying the GFZ in the initial field; if dense water is initially placed over the GFZ, it is replaced by lighter water on a time scale of less than 100 days.) This is consistent with observational evidence from floats (Jakobsen et al. 2003) and from the anomalously warm water found on the northeastern flank of the GFZ (Quadfasel and Meincke 1987), indicating a source from the East Greenland Current. The dominant larger-scale circulation feature is cyclonic flow encompassing both the Boreas Basin and the Greenland Basin. Additionally, there are closed cyclonic streamlines within each basin. There can be no EGC distinct 

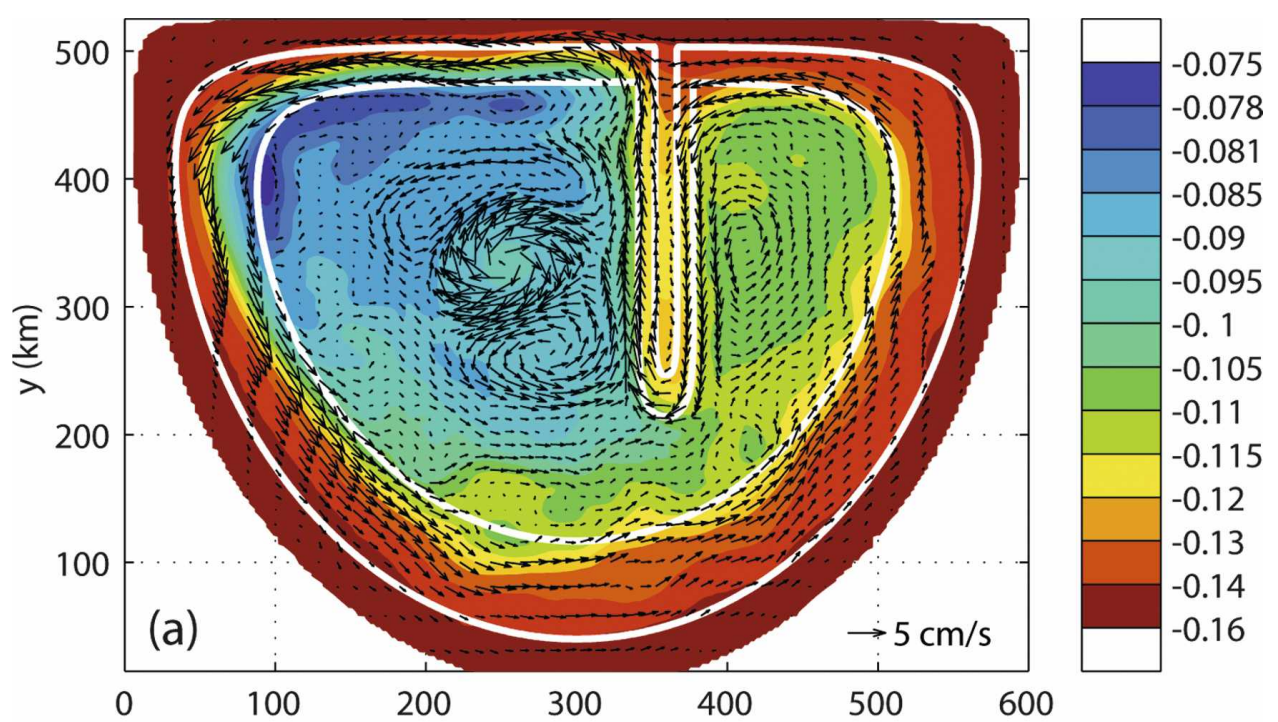

$-0.1$

$-0.105$

$-0.11$

$-0.115$

$-0.12$

$-0.13$

$-0.14$

(a)

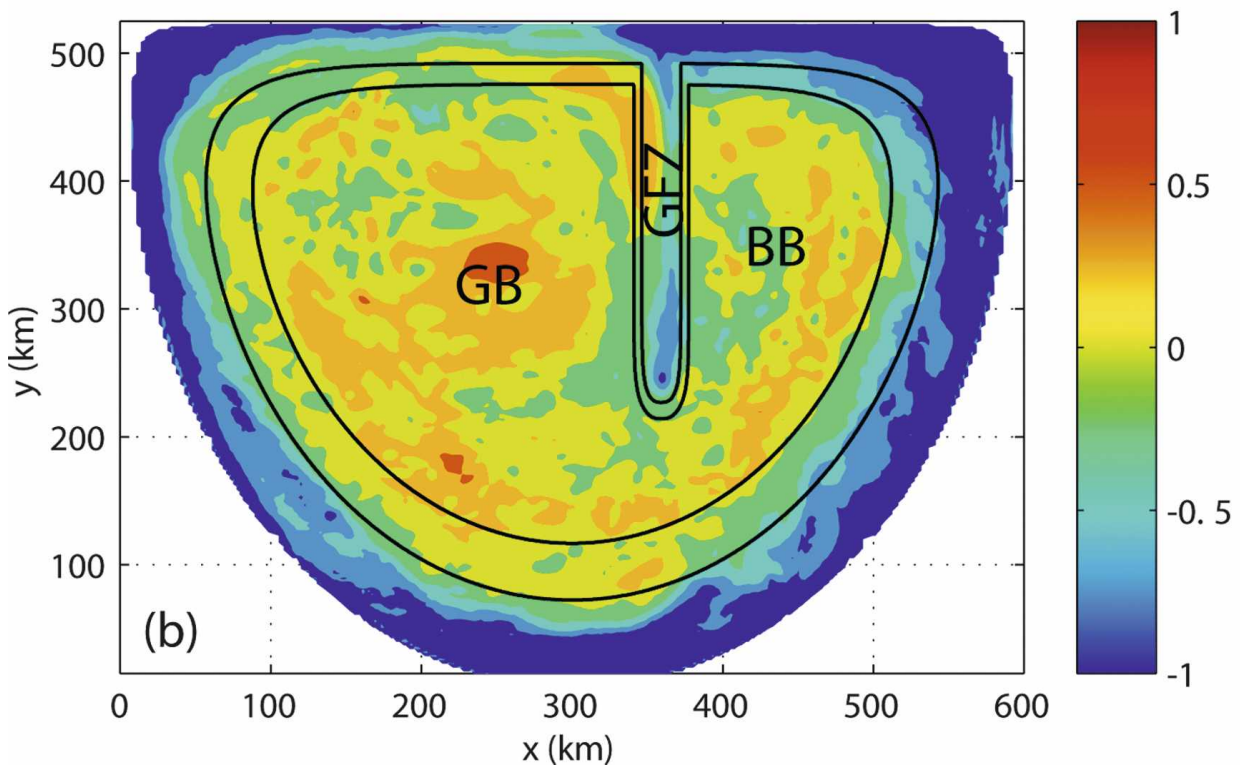

FIG. 7. The 200-400-day fields of (a) mean density, with velocity vectors (b) EKE $\left(\mathrm{cm}^{2} \mathrm{~s}^{-2}\right.$; $\log$ scale) in the idealized bathymetry basin control experiment at $1250-\mathrm{m}$ depth. Density is relative to the densest water in the basin. Velocity vectors are plotted at $1 / 20$ of the resolution of the grid in both dimensions. (a) The 2000- and 3000-m isobaths are plotted in white; (b) 2500-and 3000-m isobaths are plotted in black. (b) The representation of the Greenland Basin (GB), the Boreas Basin (BB), and the GFZ; the direction of north has been rotated so that the Greenland continental slope is at the upper limit of the plot.

from the Greenland Sea gyre, because of the closed boundaries used. Possibly associated with this, the Greenland Sea gyre center is displaced toward Greenland and away from the GFZ, if compared with observations (Fig. 1). The location of the fronts is sensitive to the exact choice of initial field and varies temporally in the real Greenland Sea as well (Budéus et al. 1993). The model also produces a mean broad anticyclone near the center of the Greenland Basin, which is a strong feature. Although float data (Poulain et al. 1996) and current observations (Oliver and Heywood 2003) suggest that the local flow often opposes the largerscale mean circulation, it is doubtful that such a strong mean feature exists in the real Greenland Sea. Figure $7 \mathrm{~b}$ shows that EKE is greatest where there are strong density gradients and weak topographic gradients. Despite strong density gradients at the GFZ, steep topography there stabilizes the flow. 


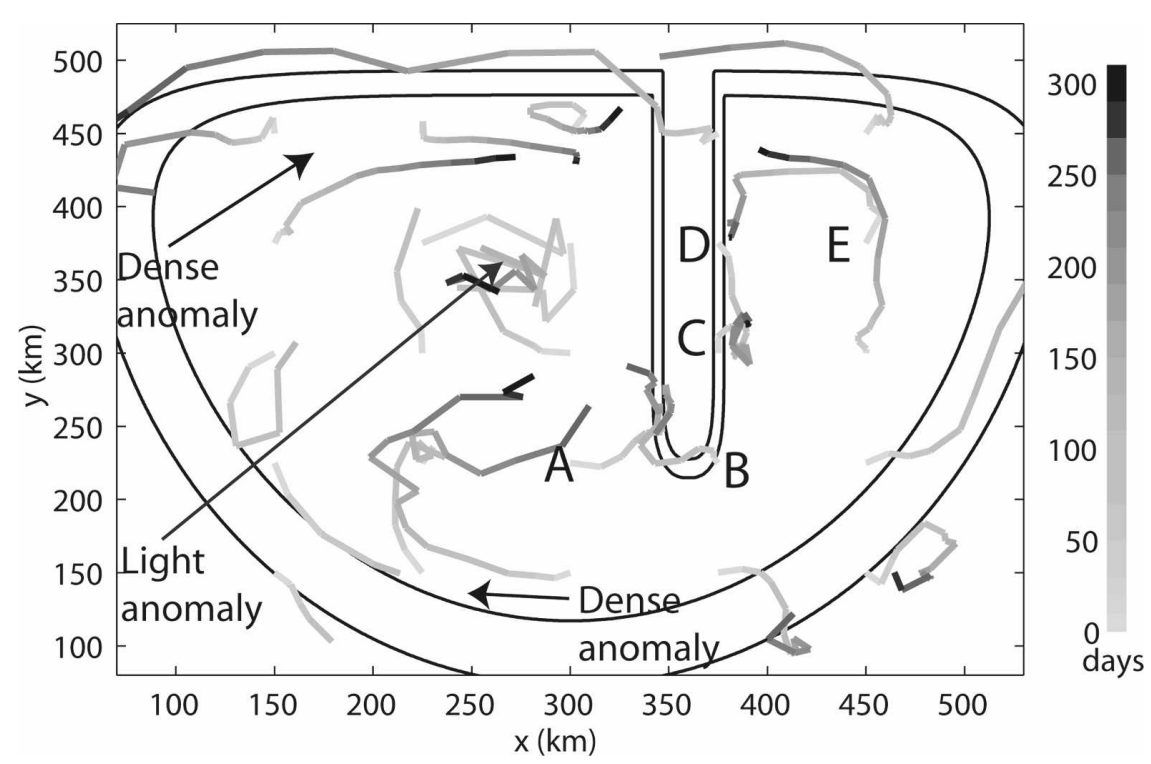

FIG. 8. Spaghetti plot of anticyclone trajectories in the idealized bathymetry basin mixedpatch release experiment. Shading indicates number of days since the release of mixed patches. Some anticyclones lose coherence before the end of the experiment. The 2500- and $3000-\mathrm{m}$ isobaths are plotted in black. Anticyclones that accumulate at the GFZ are labeled next to the start of their trajectories. Notable larger-scale features are also labeled, where the horizontal density gradient is reversed relative to the general increase in density toward the geographical gyre center (see Fig. 7).

\section{3) Vortex PROpagation AND THE ROLE OF THE GREENLAND Fracture ZoNE}

We now present results in which the control simulation is seeded with a grid of 25 mixed patches. Figure 8 is a spaghetti diagram of the trajectories of the anticyclonic component of hetons formed from these mixed patches, both before and after heton separation (note that day 0 is the time of release of the mixed patches, equivalent to day 200 in the control experiment). The majority of vortices initially propagates in a cyclonic direction around the gyre, but with a component away from the gyre center. However, some vortices are initially within, or close to, anticyclonic features, such as the large anticyclone in the center of the Greenland Sea. These propagate in an anticyclonic direction and toward the center of the anticyclonic feature. These results are consistent with advection by the ambient flow and propagation toward lighter water, as obtained in the channel experiments.

The process of propagating toward lighter water is further illustrated in Fig. 9. This shows time series of the density at $400 \mathrm{~m}$ above each of 25 anticyclones. The initial rapid decrease in density is caused primarily by sinking of the mixed patch and separation of the cyclone after release and partly by initial self-propagation. Therefore, each time series is referenced to the density at day 20 to emphasize later evolution that is affected only by propagation into waters of different density. Decreasing density at $400 \mathrm{~m}$ above the anticyclones indicates that anticyclones propagate into lighter ambient water. The channel experiments predict that the rate of propagation into lighter water is affected by the magnitude of the ambient density gradient and by the presence of topography. This is consistent with Fig.

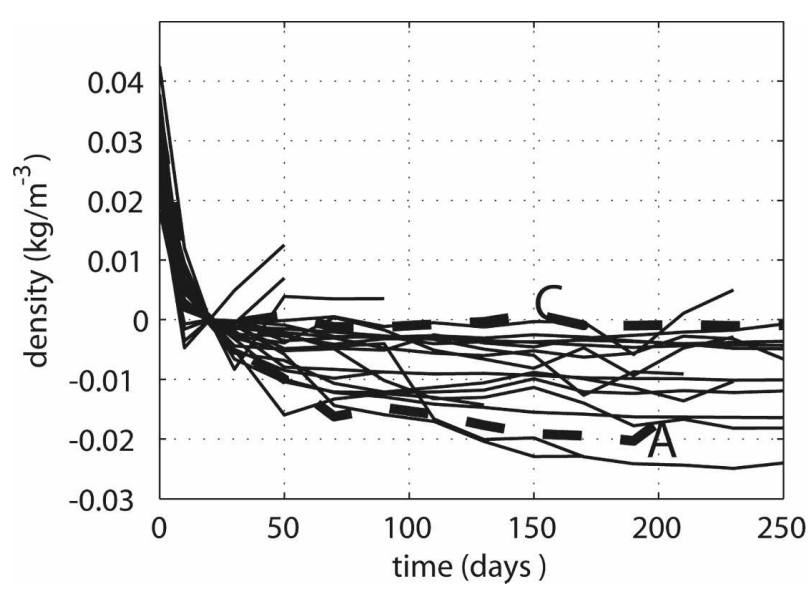

FIG. 9. Evolution of density above each anticyclone at $400 \mathrm{~m}$, referenced to density at day 20 (after initial adjustment is complete), in the idealized bathymetry basin experiment. Incomplete tracks indicate that the anticyclone lost coherence. Anticyclones A and C (see Fig. 8) are plotted with bold dashed lines. 
9, which shows that the rate at which anticyclones move into lighter water is highly variable and in some cases very small due to weak ambient gradients. The change in ambient density is also small when vortices encounter topography; the highlighted examples of anticyclones A and C, marked in Figs. 8 and 9, show that the density above these anticyclones changes little after they reach the GFZ. Nevertheless, of the 25 anticyclones released, the only four that move into denser water do so while in the process of losing coherence. All vortices that retain coherence move into lighter water.

From these results, we conclude that the anticyclonic component of a convectively formed heton in a cyclonic gyre will propagate out of that gyre unless it is trapped within a larger-scale anticyclone feature or by topography. In the Greenland Sea, the Greenland continental slope and the GFZ are the two main topographic barriers, and vortices that reach the Greenland continental slope are likely to be swept out of the gyre in the EGC. Figure 8 suggests that the GFZ catches the anticyclonic component of hetons that would otherwise depart the gyre. The tendency for anticyclones to accumulate near the GFZ is exacerbated by the longevity of anticyclones that reach the GFZ. Anticyclones in regions of high EKE (Fig. 7b) are more likely to lose coherence than those in low EKE regions such as the GFZ. Upon reaching the GFZ, some vortices are advected around the GFZ by the ambient flow (anticyclones B, D, and E in Fig. 8). However, anticyclone A remains almost stationary for 140 days until it interacts with B and both vortices lose coherence. Anticyclone $\mathrm{C}$ remains almost stationary from day 20 until the end of the simulation (day 300), merging with anticyclone D around day 120. Both of these stationary anticyclones are found within anticyclonic meanders in the ambient flow, which are mean features in the control experiment (Fig. 7).

\section{4) ReAlistiC BATHYMETRY EXPERIMENTS}

Figure 10a shows the mean density field in the realistic bathymetry control experiment. Most features are in agreement with the idealized bathymetry experiment, although the anticyclone at the center of the Greenland Sea is a much weaker feature. Another notable difference is that density is not as clearly defined by bathymetry at the GFZ, allowing the mean flow to cross the GFZ before reaching its southern tip. This is probably because the GFZ is not a continuous topographic feature at its maximum amplitude, which is how it is represented in the idealized bathymetry. However, it does not follow that the importance of the GFZ is exaggerated in the idealized bathymetry experiment and correctly diagnosed in the realistic bathymetry ex- periment. The flow around and over the GFZ is dependent on the relative importance of barotropic and baroclinic flows, which are not well constrained by observations. The limited existing evidence, summarized in Fig. 1 , suggests that the GFZ is a greater obstacle to the mean flow than obtained in the realistic bathymetry experiment. As with the idealized basin experiment, the center of the cyclonic gyre is farther from the GFZ than observations suggest, which increases the length of closed streamlines around the gyre.

Figure 10b shows anticyclone trajectories in the mixed-patch release experiment. Again, most of the features of the idealized bathymetry experiment are repeated here, with propagation into lighter water and a tendency for anticyclones to exit the gyre. Accumulation at the GFZ also occurs. Anticyclone F merges with $\mathrm{G}$, and anticyclone $\mathrm{H}$ merges with $\mathrm{I}$, and these vortices become trapped on the western flank of the GFZ. The region where these vortices accumulate is slightly to the north of where the mean flow begins to cross the GFZ in the simulation, and $\sim 80 \mathrm{~km}$ north of the location where, in reality, the greatest number of SCVs has been observed. This is the site of a preexisting larger-scale anticyclonic meander, a feature common to trapped anticyclones in the idealized bathymetry experiment and channel experiment. Once inside such a meander, an anticyclone cannot migrate downstream of the meander without either climbing bathymetry or propagating into denser water, whereas we have found that anicyclones propagate into lighter water but that this propagation is halted by steep slopes. We also note that trapping is found only where there is steep topography $\left[\beta_{H}>3 \times\right.$ $10^{-9}(\mathrm{~m} \mathrm{~s})^{-1}$ ] immediately upslope of the vortex, in both channel and basin experiments. Insofar as such scenarios are comparable with vortex-wall interactions, this would be expected to promote propagation to the right of the upslope direction (Dewar 2002b) against the ambient flow.

\section{5) Sorting of CyClones AND ANTICyClOnes}

The separation of cyclones and anticyclones upon interaction with topography, followed by the return of cyclones to denser water, is a remarkably consistent feature in both channel and basin experiments. This occurs whether the heton approaches a large vertical step, a steep slope, or a gradual slope. Identified exceptions, where the heton remains unseparated despite retardation of heton propagation by topography, are rare and limited to cases in which density gradients are weak and therefore heton propagation is slow, such as the $S_{\rho}=1 / 2$ case in Fig. 5c. (Note, however, that heton separation may also occur without apparent interaction with topography, such as in the $S_{\rho}=4$ case in Fig. 5a.) 

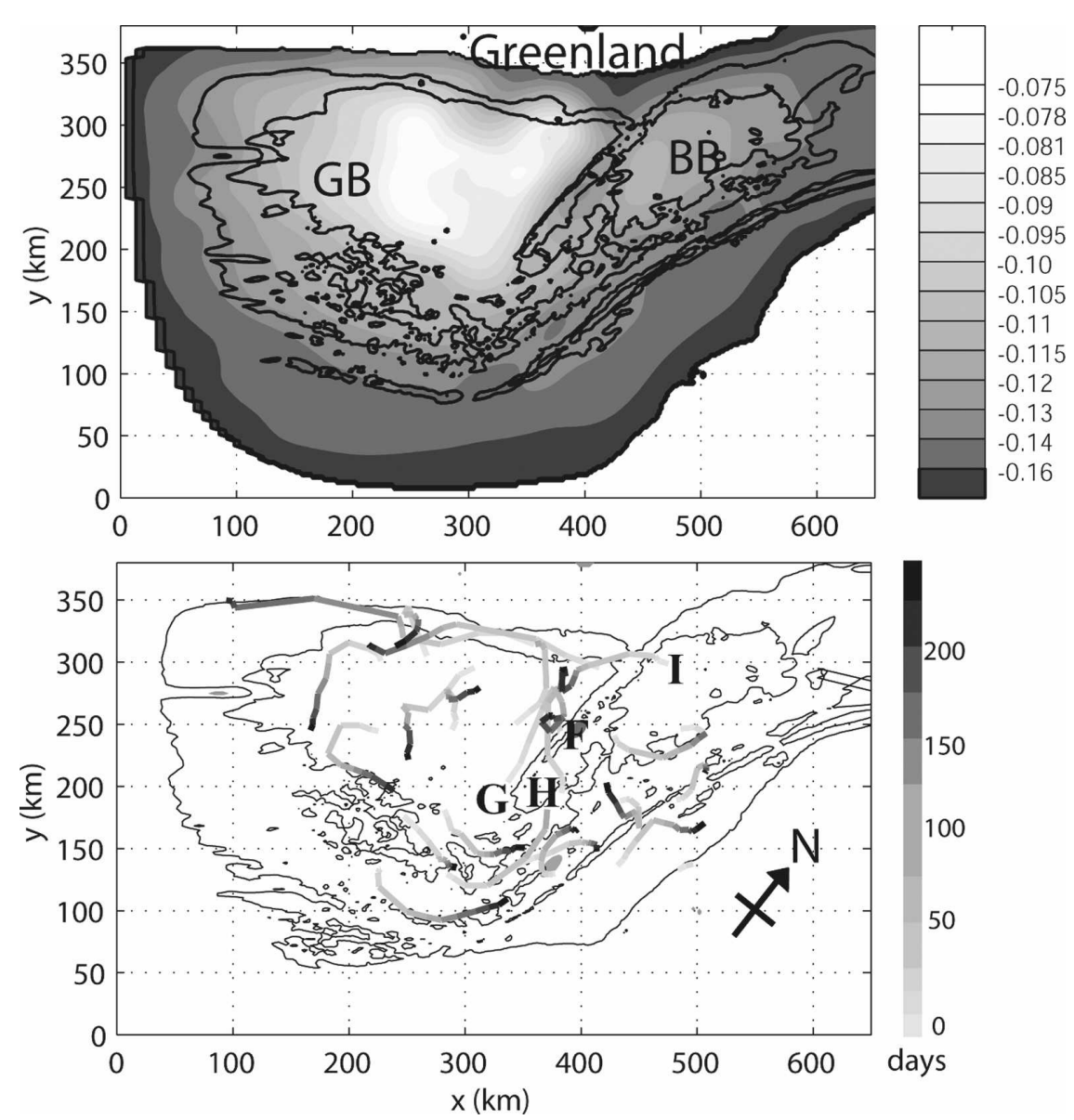

FIG. 10. (a) The 200-400-day-mean density field in the realistic bathymetry basin control experiment at $1250-\mathrm{m}$ depth. Density is relative to the densest water in the basin. The 2500and 3000-m isobaths are plotted in black. (b) Spaghetti plot of anticyclone trajectories in the realistic bathymetry mixed-patch release basin experiment. Shading indicates the number of days since the release of mixed patches. Some anticyclones lose coherence before the end of the experiment. Anticyclones that become trapped at the GFZ are labeled in bold type next to the start of their trajectories.

The initial stage of this process, heton separation, is consistent with the skewing of the vorticity field due to the control of topographic beta on potential vorticity. Dewar (2002b) argued that this process suppressed heton formation over a slope in quasigeostrophic experiments with uniform ambient density. Dewar, however, did not obtain downslope propagation of cyclones.

To understand the behavior of the lone vortices after separation, we consider the propagation of asymmetric monopolar vortices. Walsh (1995), using a quasigeostrophic model in the context of "Meddies," found that tilting of vortices due to the ambient velocity shear can cause self-propagation of vortices, provided there is also a vertical gradient in the vorticity of the vortex. This is because the tilting creates a mean horizontal asymmetry with, for example, the stronger vorticity aloft unaligned with the weaker vorticity below. The residual vorticity field, after the dominant symmetric component is removed, is similar to a baroclinic dipole. A barotropic residual dipole may also exist due to horizontal asymmetries in the vortex. Such a vortex is termed a "quasi monopole" (Stern and Radko 1998) and is sketched in Fig. 11a.

Here, we examine the residual vorticity field of 38 anticyclones and 26 cyclones, taken from all available experiments, in a vertical plane parallel with isobaths. Vortices were chosen that could clearly be identified as having recently separated (defined as a distance of $>25$ $\mathrm{km}$ between the centers of the anticyclones and cyclones) from their anticyclonic partner, following interaction with topography. Figures $11 \mathrm{~b}, \mathrm{c}$ show composite residual vorticity fields for the cyclones and anticyclones, respectively. The composites were obtained by rotating in the vertical axis of each vortex so that up- 

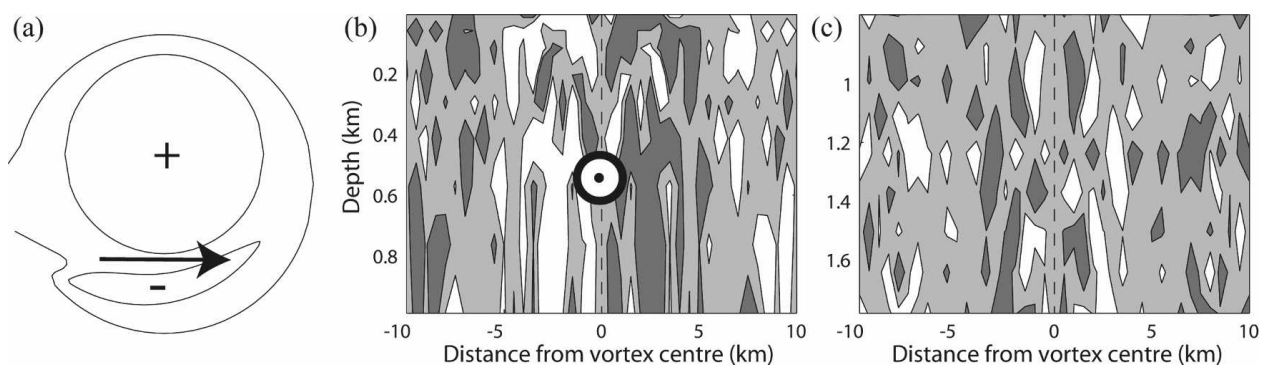

FIG. 11. (a) Plan view schematic of a quasi monopole (after Voropayev et al. 1999). The arrow shows the direction of self-propagation. (b) Vertical section of the asymmetric relative vorticity $\zeta$ component (residual after symmetric component removed) through a composite of 26 surface cyclones that have recently separated from subsurface anticyclones upon interaction with topography. Section is viewed looking upslope. Dark shading indicates $\zeta>0.3 \times 10^{-5}$; light shading indicates $0.3 \times 10^{-5}>\zeta>$ $-0.3 \times 10^{-5}$; white shading indicates $\zeta<-0.3 \times 10^{-5}$. Implied dipole propagation, due to vortex asymmetry, is downslope (toward the reader). (c) Same as in (b), but for a composite of 38 anticyclones.

slope is into the page. The mean asymmetry in the composite anticyclone, with positive vorticity to the left of the vortex center, would indicate upslope propagation, but spatial variability dominates this signal. However, the mean cyclone exhibits a coherent asymmetric component consistent with downslope propagation. (Individual cyclones do not always exhibit such asymmetry, and there were approximately four cases of asymmetry of the opposite sign.) The asymmetry is principally barotropic rather than baroclinic, suggesting that topographic rather than vertical shear effects are responsible. In laboratory tank experiments, Voropayev et al. (1999) found that a quasi monopole will propagate away from a wall following a collision, due to rearrangement of vorticity within the vortex. It is surprising, however, that the clearest asymmetry in our experiments is to be found in the surface cyclone rather than in the subsurface anticyclone, which is more directly influenced by topography.

\section{Concluding remarks}

This study provides evidence that the ambient hydrography and topography are key controls on the propagation of postconvective vortices and the associated transport of convected waters. Ambient density gradients determine the direction of self-propagation of hetons, by controlling the relative positions of their cyclonic and anticyclonic components. Thus, in our simulations, hetons move toward lighter water and typically out of gyres that are hosts to convection, providing a mechanism for removing convected water from convective regions. This propagation is arrested at steep topography, where subsurface anticyclones accumulate. A subset of anticyclones that reach such topography becomes trapped within anticyclonic meanders in the mean flow; these remain stationary and coherent for several hundred days. The cyclonic components of hetons do not accumulate at topography but return to denser waters after heton separation.

Several simplifying assumptions have been made in this study. Most importantly, the roles of wind and ongoing surface buoyancy forcing have been excluded. The significant nonlinearity of the equation of state in the Greenland Sea has also been neglected. Furthermore, the experiment setup minimizes the impact of convection on the ambient density field. Large-scale convective patches would be expected to generate strong baroclinic rim currents, which are likely to contribute to the propagation of hetons away from sites of convection (Legg and Marshall 1993). Nevertheless, the experiments are sufficiently sophisticated such that existing analytical theories cannot be used to quantitatively predict the results. The behavior of hetons under scenarios differing greatly from the presented experiments is therefore uncertain. For example, the propagation of hetons formed after full-depth convection has not been investigated. On the other hand, when mixed patches with horizontal scales exceeding the local Rossby radius were released (not shown), the mechanisms discussed here applied after the mixed patches divided into multiple hetons. A small number of experiments (not presented here) were conducted in the setting of stronger vertical stratification. The Gryanik et al. (2000) theory predicts that self-propagation speed has a weak dependence on ambient vertical density stratification (varying as $N^{-1}$ if convection depth is constant). Instead, we found that self-propagation into lighter waters was almost negligible in high stratification experiments.

With these caveats in mind, we consider the likely implications of our findings on the interpretation of 
observations in convective regions. Testor and Gascard (2006) observed the spreading phase of deep convection by following floats inside postconvective vortices. Floats in the North Balearic Front were advected rapidly by the ambient flow, but there was a component of propagation toward warmer waters (their Fig. 15), whereas floats nearer steep topography propagated more slowly or not at all. These results are consistent with the mechanisms described in this study, although the preponderance of anticyclones observed by Testor and Gascard (2006) suggests that many of the propagating anticyclones may not have been part of heton pairs. In the Greenland Sea, SCVs have been observed in the highest concentrations at the rim of the gyre, particularly at the GFZ (Gascard et al., 2002; Wadhams et al. 2004; Kasajima et al. 2006). Our results indicate that this is not strong evidence that the SCVs were initially produced by convection close to the GFZ, because anticyclones formed closer to the gyre center may also accumulate at the GFZ. High concentrations of weakly stratified SCVs at the GFZ may rather precondition the region for deep convection: convection has been observed within a preexisting SCV at the GFZ (Wadhams et al. 2004). Observations also show that at least two SCVs have become trapped close to $75^{\circ} \mathrm{N}, 0^{\circ}$ (Gascard et al. 2002), leading to speculation that the local topography favors the trapping of SCVs. In our simulations, some anticyclones were trapped within larger-scale anticyclonic meanders near the GFZ. The trapped SCV observed by Gascard et al. (2002) appears to be located within a larger- scale anticyclonic feature (indicated by minima in the depth of isopcyncals immediately outside the SCV in Fig. 3 of Gascard et al.). Other SCV surveys have not covered a large enough area to test this hypothesis. In the idealized bathymetry basin experiment, one anticyclone became trapped in a position analagous to $75^{\circ} \mathrm{N}, 0^{\circ}$ immediately downstream of the tip of the GFZ, but this was not reproduced in the realistic bathymetry basin experiment. A more robust result is that the site of an already trapped anticyclone can interact with further anticyclones that are advected around the GFZ. In our experiments, there is one example of vortex dissipation and three examples of merging as a result of such interactions, suggesting that the site of a trapped SCV is a favorable location for the trapping of further SCVs.

Acknowledgments. This study was supported by a UK NERC Rapid Programme Grant (NER/T/S/2002/ 00446) and by Norwegian Research Council project ProClim (155923/700). We thank Igor Esau, Jonathan Lilly, and Fiamma Straneo for discussion on the manuscript.

\section{REFERENCES}

Bönisch, G., J. Blindheim, J. L. Bullister, P. Schlosse, and D. W. R. Wallace, 1997: Long-term trends of temperature, salinity, density, and transient tracers in the central Greenland Sea. J. Geophys. Res., 102, 18 553-18 571.

Budéus, G., A. A. Maul, and G. Krause, 1993: Variability in the Greenland Sea as revealed by a repeated high-spatialresolution conductivity-temperature-depth survey. J. Geophys. Res., 98, 9985-10000.

— B. Cisewski, S. Ronski, D. Dietrich, and M. Weitere, 2004: Structure and effects of a long lived vortex in the Greenland Sea. Geophys. Res. Lett., 31, L05304, doi:10.1029/ 2003 GL017983.

Carnevale, G. F., R. C. Kloosterziel, and G. J. F. van Heijst, 1991: Propagation of barotropic vortices over topography in a rotating tank. J. Fluid Mech., 233, 119-139.

Carton, X., 2001: Hydrodynamical modeling of oceanic vortices. Surv. Geophys., 22, 179-263.

Clark, R. A., and J.-C. Gascard, 1983: The formation of Labrador Sea Water. Part II: Mesoscale and smaller-scale processes. $J$. Phys. Oceanogr., 13, 1779-1797.

Dewar, W. K., 2002a: Baroclinic eddy interaction with isolated topography. J. Phys. Oceanogr., 32, 2789-2805.

— 2002b: Convection in small basins. J. Phys. Oceanogr., 32, $2766-2788$.

Eldevik, T., F. Straneo, A. B. Sand $\varnothing$, and T. Furevik, 2005: Pathways and export of Greenland Sea Water. The Nordic Seas: An Integrated Perspective-Oceanography, Climatology, Biogeochemistry, and Modeling, Geophys. Monogr. Vol. 158, Amer. Geophys. Union, 89-104.

Fahrbach, E., J. Meincke, S. Østerhus, G. Rohardt, U. Schauer, V. Tcerberg, and J. Verduin, 2001: Direct measurements of heat and mass transport through Fram Strait. Polar Res., 20, $217-$ 224.

Flór, J. B., and I. Eames, 2002: Dynamics of monopolar vortices on a topographic beta-plane. J. Fluid Mech., 456, 353-376.

Furevik, T., and J. E. O. Nilsen, 2005: Large-scale atmospheric circulation variability and its impacts on the Nordic Seas ocean climate-A review. The Nordic Seas: An Integrated Perspective, Geophys. Monogr., Vol. 158, Amer. Geophys. Union, 105-136.

Gascard, J. C., A. J. Watson, M. J. Messias, K. A. Olsson, T. Johannessen, and K. Simonsen, 2002: Long-lived vortices as a mode of deep ventilation in the Greenland Sea. Nature, 416, $525-527$.

Griffiths, R. W., and E. J. Hopfinger, 1986: Experiments with baroclinic vortex pairs in a rotating fluid. J. Fluid Mech., 173, 501-518.

Gryanik, V. M., T. N. Doronina, D. J. Olbers, and T. H. Warncke, 2000: The theory of three-dimensional hetons and vortexdominated spreading in localized turbulent convection in a fast rotating stratified fluid. J. Fluid Mech., 423, 71-125.

Hogg, N. G., and H. M. Stommel, 1985: The heton, an elementary interaction between discrete baroclinic geostrophic vortices, and its implications concerning eddy heat-flow. Proc. Roy. Soc. London, 397, 1-20.

IOC, IHO, and BODC, 2003: Centenary Edition of the GEBCO Digital Atlas. General Bathymetric Chart of the Oceans (GEBCO) digital atlas, centenary edition, 1903-2003, Inter- 
governmental Oceanographic Commission and the International Hydrographic Organization, British Oceanographic Data Centre, CD-ROM. [Available online at http://www. bodc.ac.uk/projects/international/gebco/gebco-digital-atlas/.]

Jacob, J. P., E. P. Chassignet, and W. K. Dewar, 2002: Influence of topography on the propagation of isolated eddies. J. Phys. Oceanogr., 32, 2848-2868.

Jakobsen, P. K., M. H. Ribergaard, D. Quadfasel, T. Schmith, and C. W. Hughes, 2003: Near-surface circulation in the northern North Atlantic as inferred from Lagrangian drifters: Variability from the mesoscale to interannual. J. Geophys. Res., 108, 3251, doi:10.1029/2002JC001554.

Johannessen, O. M., K. Lygre, and T. Eldevik, 2005: Convective chimneys and plumes in the Northern Greenland Sea. The Nordic Seas: An Integrated Perspective, Geophys. Monogr., Vol. 158, Amer. Geophys. Union, 251-272.

Kasajima, Y., K. A. Olsson, T. Johannessen, M.-J. Messias, E. Jeansson, R. G. J. Bellerby, and I. Skjelvan, 2006: A submesocscale coherent eddy in the Greenland Sea in 2003. J. Geophys. Res., 111, C07013, doi:10.1029/2005JC003130.

Killworth, P. D., 1979: On "chimney" formation in the ocean. $J$. Phys. Oceanogr., 9, 531-554.

LaCasce, J. H., 1998: A geostrophic vortex over a slope. J. Phys. Oceanogr., 28, 2362-2381.

Legg, S., and J. Marshall, 1993: A heton model of the spreading phase of open-ocean deep convection. J. Phys. Oceanogr., 23, 1040-1056.

—, and —, 1998: The influence of the ambient flow on the spreading of convected water masses. J. Mar. Sci., 56, 107139.

- - H. Jones, and M. Viscbeck, 1996: A heton perspective of baroclinic eddy transfer in localized open ocean convection. J. Phys. Oceanogr., 26, 2251-2266.

Lilly, J. M., P. B. Rhines, F. S. Schott, K. Lavendar, J. Lazier, U. Send, and E. D'Asaro, 2003: Observations of the Labrador Sea eddy field. Prog. Oceanogr., 59, 75-176.

Marshall, J., and F. Schott, 1999: Open-ocean convection: Observations, theory, and models. Rev. Geophys., 37, 1-64.

—, C. Hill, L. Perelman, and A. Adcroft, 1997: Hydrostatic, quasi-hydrostatic, and nonhydrostatic ocean modeling. $J$. Geophys. Res., 102, 5733-5752.

Mauritzen, C., 1996: Production of dense overflow waters feeding the North Atlantic across the Greenland-Scotland Ridge. Part 2: An inverse model. Deep-Sea Res. I, 43, 807-836.

McWilliams, J. C., 1985: Submesocale, coherent vortices in the ocean. Rev. Geophys., 23, 165-182.

Messias, M.-J., and Coauthors, 2008: The Greenland Sea Tracer Experiment 1996-2002: Horizontal mixing and transport of Greenland Sea Intermediate Water. Prog. Oceanogr., 78, 85105, doi:10.1016/j.pocean.2007.06.005.

Narimousa, S., 1998: Turbulent convection into a linearly stratified fluid: The generation of subsurface anticyclones. J. Fluid Mech., 354, 101-121.

Naveira Garabato, A. C., K. I. C. Oliver, A. J. Watson, and M. J. Messias, 2004: Turbulent diapycnal mixing in the Nordic seas. J. Geophys. Res., 109, C12010, doi:10.1029/2004JC002411.

Nøst, O. A., and P. E. Isachsen, 2003: The large-scale time-mean ocean circulation in the Nordic Seas and Arctic Ocean estimated from simplified dynamics. J. Mar. Res., 61, 175-210.
Oliver, K. I. C., and K. J. Heywood, 2003: Heat and freshwater fluxes through the Nordic Seas. J. Phys. Oceanogr., 33, 10091026.

Olsson, K. A., E. Jeansson, T. Tanhua, and J. C. Gascard, 2005: The East Greenland Current studies with CFCs and released sulphur hexafluoride. J. Mar. Syst., 55, 77-95.

Pickart, R. S., W. M. Smethie, J. R. Lazier, E. P. Jones, and W. J. Jenkins, 1996: Eddies of newly formed upper Labrador Sea water. J. Geophys. Res., 399, 20 711-20 726.

Poulain, P.-M., A. Warn-Varnas, and P. P. Niiler, 1996: Nearsurface circulation of the Nordic seas as measured by Lagrangian drifters. J. Geophys. Res., 101, 18 237-18 258.

Quadfasel, D., and J. Meincke, 1987: Note on the thermal structure of the Greenland Sea gyres. Deep-Sea Res., 34, 18831888 .

Ronski, S., and G. Budeus, 2005: Time series of winter convection in the Greenland Sea. J. Geophys. Res., 110, C04015, doi:10.1029/2004JC002318.

$\longrightarrow$, and — 2006: Vertical structure reveals eddy lifetime in the Greenland Sea. J. Geophys. Res., 33, L11602, doi:10.1029/ 2006 GL026045.

Send, U., and J. Marshall, 1995: Integral effects of deep convection. J. Phys. Oceanogr., 25, 855-872.

Spall, M. A., 2004: Boundary currents and watermass transformation in marginal seas. J. Phys. Oceanogr., 34, 1197-1213.

— , and D. C. Chapman, 1998: On the efficiency of baroclinic eddy heat transport across narrow fronts. J. Phys. Oceanogr., 28, 2275-2287.

Stern, M. E., and T. Radko, 1998: The self-propagating quasimonopolar vortex. J. Phys. Oceanogr., 28, 22-39.

Testor, P., and J.-C. Gascard, 2006: Post-convection spreading phase in the Northwestern Mediterranean Sea. Deep-Sea Res., 53, 869-893.

Visbeck, M., J. Marshall, and H. Jones, 1996: Dynamics of isolated convective regions in the ocean. J. Phys. Oceanogr., 26, 17211734.

Voropayev, S. I., G. B. McEachern, D. L. Boyer, and H. J. S. Fernando, 1999: Experiment on the self-propagating quasimonopolar vortex. J. Phys. Oceanogr., 29, 2741-2751.

Wadhams, P., G. Budéus, J. P. Wilkinson, T. Løyning, and V. Pavlov, 2004: The multi-year development of long-lived convective chimneys in the Greenland Sea. Geophys. Res. Lett., 31, L06306, doi:10.1029/2003GL019017.

Walsh, D., 1995: A model of a mesoscale lens in large-scale shear. Part I: Linear calculations. J. Phys. Oceanogr., 25, 735-746. , and L. J. Pratt, 1995: The interaction of a pair of point potential vortices in uniform shear. Dyn. Atmos. Oceans, 22, 135-160.

Watson, A. J., and Coauthors, 1999: Mixing and convection in the Greenland Sea from a tracer-release experiment. Nature, 401, 902-904.

Woodgate, R. A., E. Fahrbach, and G. Rohardt, 1999: Structure and transports of the East Greenland Current at $75^{\circ} \mathrm{N}$ from moored current meters. J. Geophys. Res., 104, 18 059-18 072.

Wu, C. C., and K. A. Emanuel, 1993: Interaction of a baroclinic vortex with background shear: Application to hurricane movement. J. Atmos. Sci., 50, 62-76. 